\title{
Correction to: Psychometric properties of the fear of food measure in Japanese women
}

\author{
Maiko Hiraide ${ }^{1} \cdot$ Takeshi Horie $^{1} \cdot$ Shu Takakura ${ }^{2} \cdot$ Tomokazu Hata $^{2} \cdot$ Nobuyuki Sudo $^{2} \cdot$ Kazuhiro Yoshiuchi $^{1}$ (i)
}

Published online: 9 February 2021

(c) Springer Nature Switzerland AG 2021

\section{Correction to: \\ Eating and Weight Disorders - Studies on Anorexia, Bulimia and Obesity (2020) https://doi.org/10.1007/s40519-020-01061-9}

\section{Correction 1}

The third paragraph of the Results

With regard to the four FOFM subscales, the Cronbach's alpha coefficients were $0.96,0.91,0.88$, and 0.93 for $\mathrm{AE}$, $\mathrm{RE}, \mathrm{AS}$, and $\mathrm{AB}, \mathrm{McD}$ onald's omega and the Cronbach's alpha coefficients were 0.96 and $0.96,0.91$ and $0.91,0.88$ and 0.88 , and 0.93 and 0.93 for $\mathrm{AE}, \mathrm{RE}, \mathrm{AS}$, and $\mathrm{AB}$, respectively (Table 3 ).

Should be replaced by

With regard to the four FOFM subscales, McDonald's omega and the Cronbach's alpha coefficients were 0.96 and 0.96, 0.91 and $0.91,0.88$ and 0.88 , and 0.93 and 0.93 for $\mathrm{AE}$, $\mathrm{RE}, \mathrm{AB}$, and AS, respectively (Table 3 ).

Correction 2

The sixth paragraph of the Results

With regard to $\mathrm{AE}$ and $\mathrm{AS}$, there was a significant main effect of group $(p<0.001)$, and scores in AN-BP $(p<0.001)$ and in BN/BED $(p<0.001)$ were significantly higher than those in AN-R.

Should be replaced by

With regard to $\mathrm{AE}$ and $\mathrm{AB}$, there was a significant main effect of group $(p<0.001)$, and scores in AN-BP $(p<0.001)$

The original article can be found online at https://doi.org/10.1007/ s40519-020-01061-9.

\section{Kazuhiro Yoshiuchi}

kyoshiuc-tky@umin.ac.jp

1 Department of Stress Sciences and Psychosomatic Medicine, Graduate School of Medicine, The University of Tokyo,

7-3-1 Hongo, Bunkyo-ku, Tokyo 113-8655, Japan

2 Department of Psychosomatic Medicine, Kyushu University Hospital, 3-1-1 Maidashi, Higashi-ku, Fukuoka 812-8582, Japan and in BN/BED $(p<0.001)$ were significantly higher than those in AN-R.

Correction 3

The sixth paragraph of the Results

With regard to $\mathrm{AB}$, there was showed a significant main effect of group ( $p=0.04)$, but there was not any significant difference in scores between each pair of the three groups.

Should be replaced by

With regard to AS, there was showed a significant main effect of group ( $p=0.04)$, but there was not any significant difference in scores between each pair of the three groups. Correction 4

Table 3 Explanatory factor analysis and internal consistency of the FOFM

\begin{tabular}{|c|c|c|c|c|c|}
\hline \multicolumn{6}{|l|}{$\begin{array}{l}\text { AS: Anxiety about eating with } \\
\text { somebody }\end{array}$} \\
\hline $\begin{array}{l}\text { I feel anxious when eating } \\
\text { around other people }\end{array}$ & 0.07 & -0.08 & 0.94 & 0.02 & 0.92 \\
\hline $\begin{array}{l}\text { I do not like to eat around } \\
\text { other people because they } \\
\text { might judge me }\end{array}$ & 0.02 & 0.05 & 0.75 & 0.07 & 0.73 \\
\hline $\begin{array}{l}\text { I do not like to eat in social } \\
\text { situations }\end{array}$ & -0.08 & 0.15 & 0.84 & -0.03 & 0.76 \\
\hline \multicolumn{6}{|l|}{$\begin{array}{l}\text { AB: Anxiety about body } \\
\text { weight and shape }\end{array}$} \\
\hline $\begin{array}{l}\text { Eating makes me anxious } \\
\text { because I am afraid I might } \\
\text { get fat }\end{array}$ & 0.09 & -0.03 & 0.04 & 0.90 & 0.95 \\
\hline $\begin{array}{l}\text { I worry that eating will make } \\
\text { me dissatisfied with my } \\
\text { body }\end{array}$ & 0.02 & 0.01 & 0.04 & 0.81 & 0.73 \\
\hline $\begin{array}{l}\text { If I do not eat much because } \\
\text { I am worried about my } \\
\text { weight }\end{array}$ & -0.01 & 0.14 & -0.01 & 0.79 & 0.76 \\
\hline
\end{tabular}


Should be replaced by

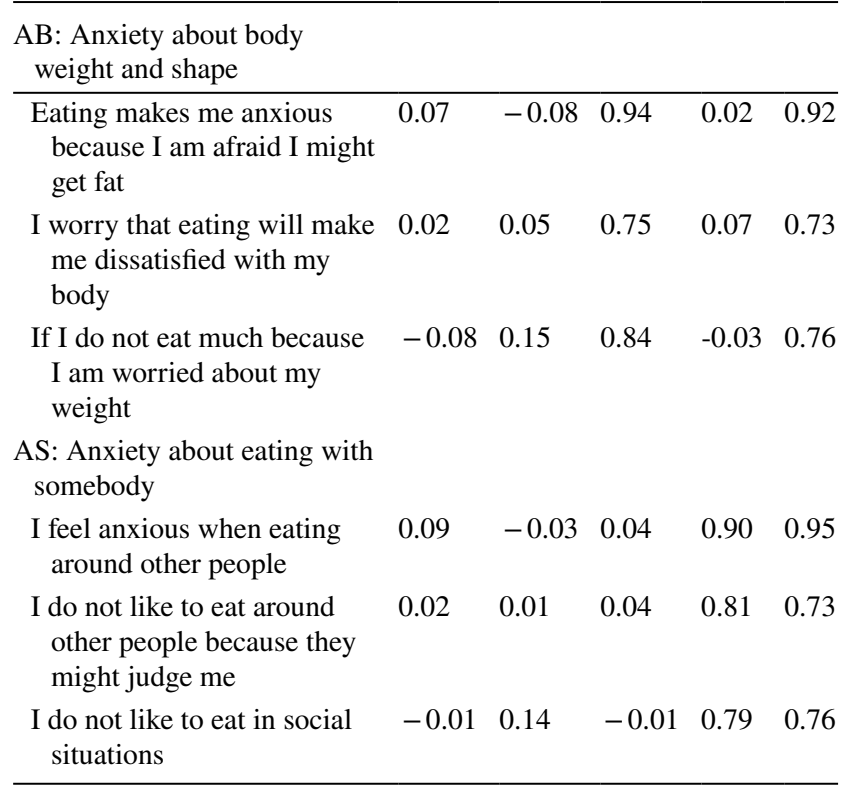

\section{Correction 5}

Table 4 Correlation coefficients between each FOFM subscale and other psychological tests

\begin{tabular}{lllll}
\hline AS & $0.47^{*}$ & $0.40^{*}$ & $0.60^{*}$ & $0.46^{*}$ \\
AB & $0.48^{*}$ & $0.41^{*}$ & $0.62^{*}$ & $0.42^{*}$ \\
\hline
\end{tabular}

Should be replaced by

Table 4 Correlation coefficients between each FOFM subscale and other psychological tests

\begin{tabular}{lllll}
\hline $\mathrm{AB}$ & $0.47^{*}$ & $0.40^{*}$ & $0.60^{*}$ & $0.46^{*}$ \\
$\mathrm{AS}$ & $0.48^{*}$ & $0.41^{*}$ & $0.62^{*}$ & $0.42^{*}$ \\
\hline
\end{tabular}

\section{Correction 6}

Table 5 Comparison between patients with eating disorders and the healthy control group

\begin{tabular}{llll}
\hline AS & $16(3-21)$ & $6(3-19)$ & $<0.001$ \\
AB & $12(3-21)$ & $3(3-21)$ & $<0.001$ \\
\hline
\end{tabular}

Should be replaced by

Table 5 Comparison between patients with eating disorders and the healthy control group

\begin{tabular}{llll}
\hline $\mathrm{AB}$ & $16(3-21)$ & $6(3-19)$ & $<0.001$ \\
$\mathrm{AS}$ & $12(3-21)$ & $3(3-21)$ & $<0.001$ \\
\hline
\end{tabular}

\section{Correction 7}

Table 6 Comparison among patients with eating disorders

\begin{tabular}{llllll} 
AS & $9.5(3-21)$ & $18(3-21)$ & $18(10-19)$ & $<0.001$ & $\begin{array}{l}\text { ANBP }>\text { ANR, } \\
\text { BN/ } \\
\text { BED }>\text { ANR }\end{array}$ \\
AB $8.5(3-21)$ & $13.5(3-21)$ & $12(3-20)$ & 0.04 & n.s \\
\hline
\end{tabular}

Should be replaced by

Table 6 Comparison among patients with eating disorders

\begin{tabular}{lccccc}
\hline AB & 9.5 & 18 & $18(10-$ & $<0.001$ & ANBP $>$ ANR, \\
& $(3-21)$ & $(3-21)$ & $19)$ & & $\begin{array}{l}\text { BN/ } \\
\text { BED }>\text { ANR }\end{array}$ \\
& & & & & n.s \\
AS & 8.5 & 13.5 & 12 & 0.04 & 12 \\
& $(3-21)$ & $(3-21)$ & $(3-20)$ & & \\
\hline
\end{tabular}

Correction 8.

Supplemental Material Table 2 The correlations among four factors

\begin{tabular}{lllll}
\hline & & & AS & AB \\
\hline & & & 0.79 & 0.78 \\
& & & 0.70 & 0.71 \\
$\mathrm{AS}$ & 0.79 & 0.70 & 1.00 & 0.66 \\
$\mathrm{AB}$ & 0.78 & 0.71 & 0.66 & 1.00 \\
\hline
\end{tabular}

Should be replaced by

Supplemental Material Table 2 The correlations among four factors

\begin{tabular}{lllll}
\hline & & & $\mathrm{AB}$ & $\mathrm{AS}$ \\
\hline & & & 0.79 & 0.78 \\
& & & 0.70 & 0.71 \\
$\mathrm{AB}$ & 0.79 & 0.70 & 1.00 & 0.66 \\
$\mathrm{AS}$ & 0.78 & 0.71 & 0.66 & 1.00 \\
\hline
\end{tabular}

Publisher's Note Springer Nature remains neutral with regard to jurisdictional claims in published maps and institutional affiliations. 